\title{
Insuficiencia renal oculta y factores asociados en pacientes con enfermedades crónicas
}

\author{
Norma Alejandra Balderas-Vargas, ${ }^{1}$ José Legorreta-Soberanis, ${ }^{2}$ Sergio Paredes-Solís, ${ }^{2 *}$ \\ Miguel Flores-Moreno, ${ }^{2}$ Felipe René Serrano-De los Santos ${ }^{2}$ y Neil Andersson ${ }^{2}$ \\ 'Instituto Mexicano del Seguro Social, Unidad de Medicina Familiar 29; '2Universidad Autónoma de Guerrero, Centro de Investigación de \\ Enfermedades Tropicales. Guerrero, México
}

\section{Resumen}

Introducción: El diagnóstico oportuno y la intervención terapéutica temprana disminuyen la mortalidad prematura asociada con insuficiencia renal crónica. Objetivo: Identificar la prevalencia y factores asociados con insuficiencia renal oculta en pacientes con enfermedades crónicas. Método: Estudio transversal de 1268 pacientes con diabetes mellitus tipo 2 e hipertensión arterial sistémica. Se usó un instrumento de medición con preguntas sobre factores asociados como artrosis, tratamiento de padecimiento crónico, tabaquismo, ingesta de analgésicos, alcoholismo, índice de masa corporal, actividad física y niveles séricos de glucosa, colesterol y triglicéridos. Resultados: La prevalencia de insuficiencia renal oculta fue de 13.2\% (167/1268), $13.4 \%$ en pacientes diabéticos (117/876) y $14.9 \%$ en hipertensos (150/1010). En el analisis multivariado, los factores asociados con insuficiencia renal oculta fueron edad > 60 años (RMa = 1.96, IC $95 \%=1.22-2.49)$, sexo femenino (RMa $=2.17$, IC $95 \%=1.30-2.82)$, padecer hipertensión arterial sistémica (RMa = 1.96, IC $95 \%=1.22-2.50)$ y no tener sobrepeso u obesidad (RMa =0.49, IC $95 \%=0.41-0.8$ ). Conclusiones: La prevalencia de insuficiencia renal oculta fue de $13 \%$. Los pacientes mayores de 60 años, con sobrepeso u obesidad e hipertensión arterial sistémica deben ser examinados detalladamente por el médico familiar para la detección temprana de insuficiencia renal oculta.

PALABRAS CLAVE: Insuficiencia renal oculta. Diabetes mellitus. Hipertensión arterial sistémica.

\section{Occult renal failure and associated factors in patients with chronic conditions}

\begin{abstract}
Introduction: Timely diagnosis and early therapeutic intervention reduce premature mortality associated with chronic renal failure. Objective: To identify the prevalence and factors associated with occult renal failure in patients with chronic diseases. Method: Crosssectional study of 1268 patients with type 2 diabetes mellitus and systemic arterial hypertension. A measuring instrument with questions about associated factors such as osteoarthritis, treatment of chronic conditions, smoking, analgesic consumption, alcoholism, body mass index, physical activity and serum glucose, cholesterol and triglyceride levels was used. Results: The prevalence of occult renal failure was $13.2 \%$ (167/1,268), $13.4 \%$ in diabetic patients (117/876) and $14.9 \%$ in hypertensive patients $(150 / 1,010)$. In the multivariate analysis, the factors associated with occult renal failure were being older than 60 years $(a O R=1.96,95 \% \mathrm{Cl}$ $=1.22-2.49$ ), belonging to the female gender $(a O R=2.17,95 \% \mathrm{Cl}=1.30-2.82)$, suffering from systemic arterial hypertension $(a O R=1.96,95 \% \mathrm{Cl}=1.22-2.50)$ and not having overweight/obesity (aOR $=0.49,95 \% \mathrm{Cl}=0.41-0.8)$. Conclusions: The prevalence of occult renal failure was $13 \%$. Female patients older than 60 years with overweight/obesity and systemic arterial hypertension should be examined in detail by the family doctor for occult renal failure early detection.
\end{abstract}

KEY WORDS: Occult renal failure. Diabetes mellitus. Systemic arterial hypertension.

Correspondencia:

*Sergio Paredes-Solís

E-mail: srgprds@gmail.com

CC BY-NC-ND (http://creativecommons.org/licenses/by-nc-nd/4.0/).
Fecha de recepción: 17-05-2019

Fecha de aceptación: 14-07-2019

DOI: $10.24875 / G M M .19005292$
Gac Med Mex. 2020;156:11-16

Disponible en PubMed

www.gacetamedicademexico.com 


\section{Introducción}

En 1990, la enfermedad renal crónica ocupó el lugar 27 en la lista de causas de mortalidad global, con tasa anual estandarizada por edad de 9.6 por 100000 habitantes, que se incrementó a 11.1 por 100000 habitantes en 2010. En el mundo se estima una prevalencia de enfermedad renal crónica de $10 \%,{ }^{2}$ enfermedad que junto con otras comorbilidades crónicas incrementa el riesgo de muerte temprana. ${ }^{3,4}$ En México, la muerte prematura por enfermedad renal crónica aumentó casi $400 \%$ de 1990 a 2010. ${ }^{5}$ No obstante lo anterior, la frecuencia de este padecimiento se desconoce; un estudio en mayores de 18 años encontró una prevalencia de $8 \%{ }^{6}$

En México, en la Encuesta Nacional de Salud y Nutrición 2012 se identificó 22.4 millones de adultos con hipertensión arterial sistémica (HAS) y 6.4 millones de adultos con diabetes mellitus tipo 2 (DM2). Las cifras son relevantes debido a que solo la mitad de las personas sabe que padece hipertensión y solo la cuarta parte de diabéticos tiene su enfermedad controlada. ${ }^{7}$ La combinación de padecimientos crónicos, con el consecuente impacto negativo para la salud individual y el alto costo social, convierte a la falla renal crónica en un evento catastrófico. ${ }^{8-10}$

La falla inicial de la función renal es resultado de interacciones complejas, principalmente de padecimientos crónicos y degenerativos. Los factores de progresión que empeoran y aceleran el daño renal son la proteinuria persistente, $,^{11} \mathrm{HAS},{ }^{12} \mathrm{DM} 2$ mal controlada,${ }^{13}$ tabaquismo,${ }^{14}$ dislipidemia,${ }^{15}$ anemia, ${ }^{16}$ enfermedad vascular asociada y obesidad. ${ }^{11}$ Hay mayor frecuencia de insuficiencia renal oculta en las mujeres $^{17,18} \mathrm{y}$ en personas mayores de 50 años. ${ }^{19}$ El índice de masa corporal $>25$ se ha asociado con insuficiencia renal oculta. ${ }^{20}$ Algunas restricciones alimentarias modifican el consumo de proteínas, sodio y fósforo y detienen el daño renal, ${ }^{21}$ con mayor beneficio si se establecen oportunamente. El objetivo de este estudio fue estimar la prevalencia de insuficiencia renal oculta y los factores asociados en pacientes con HAS - DM2 de la Unidad de Medicina Familiar 29, Instituto Mexicano del Seguro Social en Acapulco, Guerrero, México.

\section{Método}

Estudio transversal realizado en usuarios de la Unidad de Medicina Familiar 29 del Instituto Mexicano del Seguro Social, del 1 de noviembre de 2014 al 31 de enero de 2015. Para la selección de los pacientes se utilizaron los siguientes criterios:

- De inclusión: pacientes con diagnóstico clínico de HAS (tensión arterial sistólica $>140 \mathrm{~mm} \mathrm{Hg}$ y diastólica > $90 \mathrm{~mm} \mathrm{Hg}$ ) o DM2 (glucemia $>126 \mathrm{mg} / \mathrm{dL}$ ); sin diagnóstico registrado de enfermedad renal crónica.

- De exclusión: pacientes sin estudios de laboratorio recientes (niveles sanguíneos de glucosa, colesterol, triglicéridos y creatinina) o que tuvieron riñón único, algún tipo de cáncer o lupus eritematoso sistémico.

El tamaño de la muestra se calculó considerando la prevalencia de insuficiencia renal oculta de $7.6 \%,{ }^{22}$ nivel de confianza de $95 \%$ (error alfa), poder del estudio de 0.20 (error beta) y una razón de momios (RM) de 1.9 entre HAS e insuficiencia renal oculta. El resultado fue de 1268 pacientes; fueron excluidos cinco.

Para medir los potenciales factores asociados con insuficiencia renal oculta se utilizó un cuestionario de 28 preguntas para recolectar datos directamente de los pacientes. El cuestionario fue validado previamente mediante ronda de expertos: ${ }^{23}$ un médico familiar, un especialista en nefrología y dos epidemiólogos. Con el cuestionario se confirmaron los datos generales del paciente: sexo, edad, raza y motivo por el cual acudió a consulta. Se verificó el tiempo de evolución del padecimiento, tratamiento actual de la enfermedad y se preguntó sobre uso de analgésicos no esteroideos, consumo de tabaco y alcohol, artrosis y periodicidad de actividad física (ejercicio físico por al menos 30 minutos diarios, incluyendo labores domésticas). Después de la entrevista, en cada paciente se midió peso corporal, estatura y presión arterial. Con el peso corporal y la estatura se estimó el índice de masa corporal.

Del expediente clínico electrónico se tomaron los datos de niveles de concentración plasmática de colesterol y triglicéridos. Con la edad, sexo y raza del paciente, así como el resultado más reciente del nivel sérico de creatinina, se calculó la tasa de filtrado glomerular con la fórmula propuesta por el grupo Chronic Kidney Disease Epidemiology Collaboration. ${ }^{24}$

La definición operativa de insuficiencia renal oculta fue la siguiente: paciente con tasa de filtración glomerular $<60 \mathrm{~mL} /$ minuto por más de tres meses y que desconocía tener enfermedad renal crónica. Con la tasa de filtrado glomerular se clasificó el grado de severidad de la enfermedad renal crónica en los seis 
Tabla 1. Estadificación de la tasa de filtración glomerular, según la guía de Kidney Disease Outcomes Quality Initiative

\begin{tabular}{|l|c|c|c|}
\hline Etapa y definición & Tasa de filltración glomerular (mL/minuto) & $n=1268$ & $\%$ \\
\hline Sin daño renal & $>91$ & 677 \\
\hline 1 Evidencia de daño renal y TFG normal & $\geq 90$ & 17 \\
\hline 2 Evidencia de daño renal y TFG ligeramente disminuida & $60-89$ & 407 & 32.1 \\
\hline 3 Disminución moderada de la TFG & $30-59$ & 161 & 12.7 \\
\hline $\begin{array}{l}\text { 4 Dsiminución grave de la TFG } \\
\text { 5 Falla renal terminal }\end{array}$ & $15-29$ & 5 & 0.4 \\
\hline TFG = tasa de filtración glomerular. & $<15$ & 1 & 0.1 \\
\hline
\end{tabular}

estadios según la propuesta de la guía de Kidney Disease Outcomes Quality Initiative. ${ }^{24}$

El análisis de datos se hizo con el programa estadístico CIETmap. ${ }^{25}$ La asociación entre las variables incluidas en el estudio y la insuficiencia renal oculta se estimó con el proceso de Mantel-Haenszel mediante razón de momios (RM), tanto en el análisis bivariado como en el multivariado. ${ }^{26}$ El intervalo de confianza de 95 \% (IC 95 \%) de la RM se estimó con la propuesta de Miettinen. ${ }^{27}$

Todos los pacientes firmaron el consentimiento informado. La información proporcionada por los pacientes fue confidencial pero no anónima. El estudio fue autorizado por el Comité Local de Investigación 1101 del Instituto Mexicano del Seguro Social, con folio R-2014-1101-15.

\section{Resultados}

Participaron 1268 pacientes con diagnóstico clínico de DM2 o HAS y sin diagnóstico de enfermedad renal crónica; $31 \%$ (392) tuvo diagnóstico de HAS, $20 \%$ (258) de DM2 y 49 \% (618) de ambos padecimientos; $63 \%$ (794) fue del sexo femenino y el promedio de edad fue de $60.1 \pm 11.1$ años, con rango de 22 a 91 años.

El $3 \%(41)$ de los pacientes refirió realizar ejericicio físico diario, 8 \% (107) antecedente de tabaquismo, $0.3 \%$ (4) consumo de alcohol y $14 \%$ (173) padecer algún tipo de artrosis. Por el nivel de lípidos sanguíneos, los pacientes tuvieron la distribución siguiente: normal $40 \%$ (507), hipertrigliceridemia $26 \%$ (325), hipercolesterolemia $12 \%$ (151) y dislipidemia $23 \%$ (285).

El $13.2 \%$ (167) de los pacientes tuvo insuficiencia renal oculta; 53 \% (677), tasa de filtración glomerular $>91 \mathrm{~mL} /$ minuto. La distribución de la tasa de filtrado glomerular de los pacientes se muestra en la Tabla 1. En pacientes con solo DM2, la ocurrencia de insuficiencia renal oculta fue de $45 \%$ (117/258), en quienes únicamente padecían HAS fue de $38 \%$ (150/392) y en pacientes con ambas condiciones, de $16.2 \%(100 / 618)$.

En el análisis bivariado se encontró que cuatro factores estuvieron asociados significativamente con insuficiencia renal oculta: sexo femenino, edad mayor de 60 años, padecer HAS e índice de masa corporal $<25$. En la Tabla 2 se registra la fuerza de asociación y los intervalos de confianza de $95 \%$ de los factores incluidos en el análisis bivariado.

El análisis multivariado mostró efecto independiente de los cuatro factores identificados en el análisis bivariado. La edad $>60$ años tuvo la mayor fuerza de asociación ( $\mathrm{RMa}=2.63$, IC $95 \% \mathrm{a}=1.84-3.76)$ y en orden decreciente siguieron padecer HAS y ser mujer. El índice de masa corporal $<25$ mostró ser un factor protector $(\mathrm{RMa}=0.60, \mathrm{IC} 95 \% \mathrm{a}=0.88-0.41)$ contra insuficiencia renal oculta; en la Tabla 3 se muestra el modelo final del análisis multivariado.

\section{Discusión}

En este estudio se encontró prevalencia de insuficiencia renal oculta de $13.2 \%$ en pacientes con HAS o DM2. También hubo evidencia de cuatro factores asociados con insuficiencia renal oculta: sexo femenino, edad > 60 años, presencia de HAS e índice de masa corporal $<25$.

En nuestro estudio utilizamos la ecuación propuesta por Chronic Kidney Disease-Epidemiology Collaboration porque proporciona más ventajas y mayor exactitud en comparación con la propuesta por Modificacion of Diet in Renal Disease, además de que mejora la capacidad predictiva de la filtración glomerular y es útil en la predicción de mortalidad global, cardiovascular y del riesgo de presentar enfermedad renal crónica terminal. ${ }^{28}$ 
Tabla 2. Análisis bivariado de factores asociados con insuficiencia renal oculta

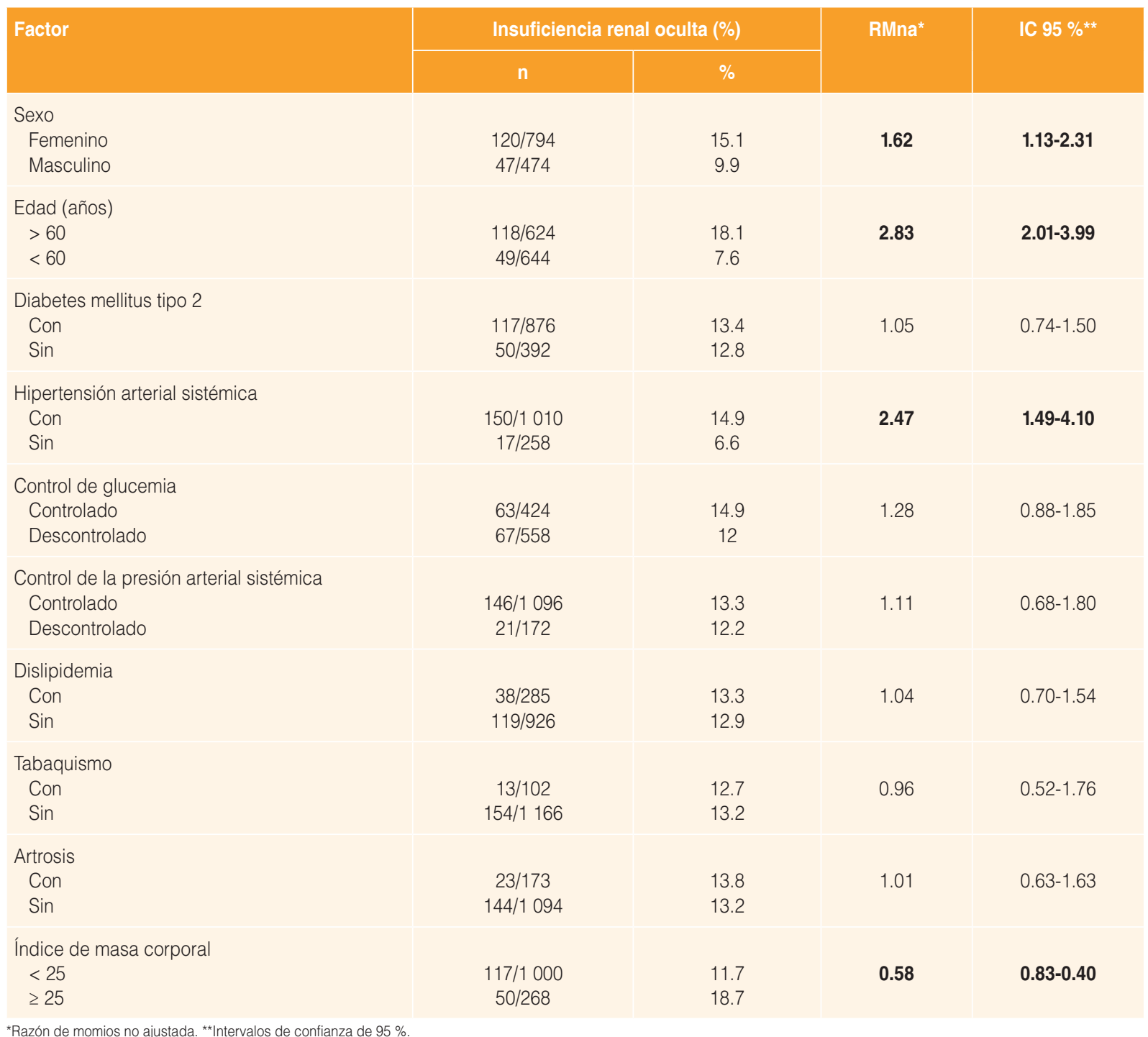

Tabla 3. Modelo final del análisis multivariado de factores asociados con insuficiencia renal oculta

\begin{tabular}{|l|c|c|c|c|c|}
\hline Factor & RMna* & RMa** & IC 95 \% $\mathbf{a}^{\mathbf{2}}$ & $\chi^{2}$ het $^{*}$ & $p$ \\
\hline Sexo femenino & 1.62 & 1.78 & $1.24-2.56$ & 9.8 & 0.53 \\
\hline $\begin{array}{l}\text { Mayor de } 60 \text { años } \\
\text { de edad }\end{array}$ & 2.83 & 2.63 & $1.84-3.76$ & 28.4 & 0.57 \\
$\begin{array}{l}\text { Hipertensión } \\
\text { arterial sistémica }\end{array}$ & 2.47 & 2.03 & $1.18-3.47$ & 6.6 & 0.67 \\
$\begin{array}{l}\text { Índice de masa } \\
\text { corporal <25 }\end{array}$ & 0.58 & 0.60 & $0.88-0.41$ & 7.0 & 0.60 \\
\hline
\end{tabular}

*Razón de momios no ajustada. **Razón de momios ajustado. ${ }^{\&}$ Intervalos de confianza de $95 \%$ de la razón de momios ajustada. "Chi cuadrada de heterogeneidad.

Encontramos que $13.2 \%$ de los pacientes con DM2 - HAS tuvo insuficiencia renal oculta, cifra cercana a $13.5 \%$, reportada por Peralta en Estados Unidos. ${ }^{8}$ En virtud de que la prevalencia en nuestro estudio se estimó en pacientes con DM2 o HAS, la ocurrencia debió estar sobrestimada porque toda la población presentaba factores de riesgo para insuficiencia renal oculta.

En nuestra investigación, el sexo femenino fue el factor con mayor fuerza de asociación con insuficiencia renal oculta, hallazgo que no es nuevo. ${ }^{17,19}$ Diversas teorías plantean que es más frecuente en mujeres debido a diferencias anatómicas con el tamaño del riñón del hombre, ${ }^{29}$ como respuesta diferenciada a angiotensina, ${ }^{30}$ estilos de vida según el género ${ }^{31} \mathrm{y}$ cambios hormonales en la mujer. ${ }^{32}$ Ser mujer y tener más de 60 años de edad son factores de riesgo para desarrollar insuficiencia renal oculta. ${ }^{33}$ También encontramos que la edad $>60$ años estuvo asociada con insuficiencia renal oculta. Otros estudios han encontrado resultados similares. ${ }^{12,18}$ 
La HAS asociada con insuficiencia renal oculta ha sido reportada por múltiples estudios observacionales que la han identificado como un factor de riesgo modificable, tanto para el desarrollo de la enfermedad renal crónica como para su progresión. ${ }^{34-37}$

Nuestro estudio reveló que el índice de masa corporal $<25$ fue un factor protector contra insuficiencia renal oculta. El mismo resultado fue reportado por Kalyesubula. ${ }^{17}$ Otero encontró una media de índice de masa corporal similar $(<27)$ como factor protector. ${ }^{15}$ Da Silva plantea la relación entre obesidad y daño renal por la acumulación de tejido adiposo, principalmente en vísceras, lo que causa compresión del riñón, con el consecuente aumento de la presión intrarrenal. También la obesidad incrementa los procesos inflamatorios y de producción de adipocitos. ${ }^{38}$

La temporalidad es uno de las principales limitaciones de los estudios transversales. En nuestro estudio es necesario discernir si el índice de masa corporal bajo es resultado de la presencia de DM2, HAS o de insuficiencia renal oculta, porque en el contexto de estos pacientes, la realización de dieta y ejercicio físico como parte del tratamiento y control de la enfermedad derivan en reducción del peso corporal. Consideramos que el índice de masa corporal alto precede a la insuficiencia renal oculta. Nuestro resultado es similar al reportado por otros estudios que también encontraron efecto protector del índice de masa corporal normal..$^{15,17}$

Es necesario capacitar y actualizar a los profesionales de la salud como parte de las mejoras al sistema sanitario, para que los médicos de primer contacto tengan la capacidad de identificar a los pacientes en etapas tempranas de la enfermedad renal. ${ }^{39}$ La detección temprana de insuficiencia renal oculta en pacientes con enfermedades crónicas es factible de implementar en el primer nivel de atención a la salud.

\section{Agradecimientos}

Agradecemos la participación de los pacientes de la Unidad de Medicina Familiar 29 del Instituto Mexicano del Seguro Social en Acapulco, Guerrero, por compartir con nosotros su experiencia con las enfermedades que les afectan.

\section{Bibliografía}

1. Lozano R, Naghavi M, Foreman K, Lim S, Shibuya K, Aboyans V, et al. Global and regional mortality from 235 causes of death for 20 age groups in 1990 and 2010: a systematic analysis for the Global Burden of Disease Study 2010. Lancet. 2012;380:2095-2128.
2. Pan American Health Organization. La OPS/OMS y la Sociedad Latinoamericana de Nefrología llaman a prevenir la enfermedad renal y a mejorar el acceso al tratamiento [en línea]. EE. UU.: Pan American Health Organization; 2018.

3. Sarnak MJ, Levey AS, Schoolwerth AC, Coresh J, Culleton B, Hamm LL, et al. Kidney disease as a risk factor for development of cardiovascular disease: a statement from the American Heart Association Councils on Kidney in Cardiovascular Disease, High Blood Pressure Research, Clinical Cardiology, and Epidemiology and Prevention. Hypertension. 2003; 42:1050-1065

4. Weiner DE, Tighiouart H, Amin MG, Stark PC, MacLeod B, Griffith JL, et al. Chronic kidney disease as a risk factor for cardiovascular disease and all-cause mortality: a pooled analysis of community-based studies. J Am Soc Nephrol. 2004;15:1307-1315.

5. Lozano R, Gómez-Dantés H, Garrido-Latorre F, Jiménez-Corona A, Campuzano-Rincón JC, Franco-Marina F, et al. La carga de enfermedad, lesiones, factores de riesgo y los desafíos para el sistema de salud en México. Salud Publica Mex. 2013:55:580-594.

6. Amato D, Álvarez-Aguilar C, Castañeda-Limones R, Rodríguez E, Ávila-Diaz M, Arreola F, et al. Prevalence of chronic kidney disease in an urban Mexican population. Kidney Int Suppl. 2005:S11-S17.

7. Instituto Nacional de Salud Pública. Encuesta Nacional de Salud y Nutrición 2012. Resultados Nacionales. México: Instituto Nacional de Salud Pública; 2012

8. Peralta CA, Muntner P, Scherzer R, Judd S, Cushman M, Shlipak MG A risk score to guide cystatin $\mathrm{c}$ testing to detect occult reduced estimated glomerular filtration rate. Am J Nephrol. 2015;42:141-147.

9. Méndez-Durán A, Méndez-Bueno JF, Tapia-Yáñez T, Muñoz-Montes A, Aguilar-Sánchez L. Epidemiología de la insuficiencia renal crónica en México. Diálisis y Trasplante. 2010;31:7-11.

10. Pereira BDS, Fernandes NDS, De Melo NP, Abrita R, Grincenkov FRDS, Fernandes NMDS. Beyond quality of life: a cross sectional study on the mental health of patients with chronic kidney disease undergoing dialysis and their caregivers. Health Qual Life Outcomes. 2017;15:74.

11. Kovesdy CP, Furth SL, Zoccali C, World Kidney Day Steering Committee. Obesity and kidney disease: hidden consequences of the epidemic. J Nephrol. 2017;27:85-92.

12. Salvador-González B, Rodríguez-Pascual M, Ruipérez-Guijarro L, Ferré-González A, Cunillera-Puertolas O, Rodríguez Latre LM. Enfermedad renal crónica en atención primaria: prevalencia y factores de riesgo asociados. Aten Primaria. 2015:47:236-245.

13. Al-Rubeaan K Yousse AM, Subhani SN, Ahma NA, Al-Sharqawi AH, Al-Mutla HM, et al. Diabetic nephropathy and its risk factors in a society with a type 2 diabetes epidemic: a Saudi National Diabetes Registry-based study. PLoS One. 2014:9:e88956.

14. Secretaría de Salud. Guía de práctica clínica. Prevención, diagnóstico y tratamiento de la enfermedad renal crónica temprana. México: Secretaría de Salud; 2009.

15. Otero A, Abelleira A, Gayoso P. Enfermedad renal crónica oculta (ERCO) y factores de riesgo vascular (FRV) asociados. Estudio epidemiológico. Nefrología. 2005;25:275-287.

16. Salvador-González B, Rodríguez-Pascual M, Ruipérez-Guijarro L, Ferré-González A, Cunillera-Puertolas O, Rodríguez-Latre LM. Chronic kidney disease in primary health care: prevalence and associated risk factors. Aten Primaria.2014:47:236-245.

17. Kalyesubula R, Nankabirwa JI, Ssinabulya I, Siddharthan T, Kayima J, Nakibuuca J, et al. Kidney disease in Uganda: a community-based study. BMC Nephrol. 2017:18:116.

18. Calvo-Vázquez I, Sánchez-Luna O, Yáñez-Sosa AL. Prevalencia de enfermedad renal crónica no diagnosticada en pacientes con diabetes meIlitus tipio 2 en atención primaria a la salud. Med Int Mex. 2015;31:41-49.

19. Rodríguez-Poncelas A, Quesada-Sabate M, Coll-De Tuero G, Caula-Ros J, Gelada-Batlle E, Gómez-Marcos MA, et al. Prevalencia de insuficiencia renal oculta y variables asociadas en una población de pacientes con diabetes tipo 2. Med Clin. 2010;134:239-245.

20. Kovesdy CP, Furth SL, Zoccali C. Obesity and kidney disease: hidden consequences of the epidemic. Braz J Med Biol Res. 2017;50:e6075.

21. Espinoza-Cuevas MA. Enfermedad renal. Gac Med Mex. 2016;152:90-96.

22. Leyva-Jiménez R, Vázquez-Aguilar LR, Alvarado-García HM. Insuficiencia renal oculta en diabéticos tipo 2 de una unidad médica de atención primaria. Aten Primaria. 2013:45:176-177.

23. Escobar-Pérez J, Cuervo-Martínez A. Validez de contenido y juicio de expertos: una aproximación a su utilización. Avances en Medición. 2008;6:27-36.

24. Montañés-Bermúdez R, Bover-San Juan J, Oliver-Samper A, Ballarín-Castán JA Gràcia-García S. Valoración de la nueva ecuación CKD-EPI para la estimación del filtrado glomerular. Nefrologia. 2010;30(2):185-94.

25. Andersson N, Mitchell S. CIETmap: free GIS and epidemiology software from the CIETgroup, helping to build the community voice into planning. Canadá: World Congress of Epidemiology; 2002.

26. Mantel N, Haenszel W. Statistical aspects of the analysis of data from retrospective studies of disease. J Natl Cancer Inst. 1959:22:719-748.

27. Miettinen OS. Simple interval estimation of the risk ratio. Am J Epidemiol. 1974;100:515-516. 
28. Martinez-Castelao A, Gorriz-Teruel JL, Bover-SanJuan J, Segura-de la Morena J, Cebollada J, Escalada J, et al. Documento de consenso sobre la enfermedad renal crónica. Nefrología. 2014;34:243-262.

29. Women's health and kidney disease. Nat Rev Nephrol. 2018;14:139.

30. Gómez-Garre D, Largo R, Ríos K, Egido J, Plaza JJ, Marrón B, et al. E sistema renina-angiotensina en la enfermedad renal progresiva. Nefrología. 1998;18:30-36.

31. Heras M, Fernández-Reyes MJ, Teresa-Guerrero M. Sobre la estimación de la función renal en el anciano: implicaciones del uso sistemático de la fórmula Modification of Diet in Renal Disease para el ajuste farmacológico. Rev Esp Geriatr Gerontol. 2010;45:49-51.

32. Goldberg I, Krause I. The role of gender in chronic kidney disease. EMJ 2016;1:58-64

33. Zhang QL, Rothenbacher D. Prevalence of chronic kidney disease in population-based studies: systematic review. BMC Public Health. 2008:8:117.

34. Sepanlou SG, Barahimi H, Najafi I, Kamangar F, Poustchi H, Shakeri R et al. Prevalence and determinants of chronic kidney disease in northeas of Iran: results of the Golestan cohort study. PLoS One. 2017;12:e0176540.
35. Levey AS, Atkins R, Coresh J, Cohen EP, Collins AJ, Eckardt KU, et al. Chronic kidney disease as a global public health problem: approaches and initiatives - a position statement from Kidney Disease Improving Global Outcomes. Kidney Int. 2007;72:247-259.

36. Klag MJ, Whelton PK, Randall BL, Neaton JD, Brancati FL, Ford CE, et al. Blood pressure and end-stage renal disease in men. $\mathrm{N}$ Engl $\mathrm{J}$ Med. 1996;334:13-18

37. Tozawa M, Iseki K, Iseki C, Kinjo K, Ikemiya Y, Takishita S. Blood pressure predicts risk of developing end-stage renal disease in men and women. Hypertension. 2003;41:1341-1345.

38. Bezerra-Da Silva JG, Novaes-Bentes ACS, De Francesco-Daher E, Alvim-De Matos SM. Obesity and kidney disease. J Bras Nefrol. 2017; 39:65-69.

39. Correa-Rotter R, González-Michaca L. Early detection and prevention of diabetic nephropathy: a challenge calling for mandatory action for Mexico and the developing world. Kidney Int Suppl. Kidney Int Suppl. 2005;(98):S69-S75. 